\title{
CAPTURA DE ENJAMBRES DE ABEJAS NATIVAS SIN AGUIJÓN CON DISPOSITIVOS - TRAMPA EN EL CAMPUS UNIVERSITARIO DEL CEAD ACACIAS - META
}

\section{CAPTURE OF NATIVE BEE SWARM WITHOUT STEMS WITH DEVICES - TRAP AT CEAD ACACIAS UNIVERSITY CAMPUS - META}

\author{
Dayro Cortes Martínez \\ Zootecnista Esp. \\ Universidad Nacional Abierta y a Distancia \\ ORCID: https://orcid.org/0000-0002-2261-3778 \\ dayro.cortes@unad.edu.co \\ Oscar Javier Olarte Blandón \\ Zootecnista Ms \\ Universidad Nacional Abierta y a Distancia \\ ORCID: https://orcid.org/0000-0003-4615-3056 \\ oscar.olarte@unad.edu.co \\ Lina Marcela Valdés Cortes \\ Estudiante de Zootecnia \\ Universidad Nacional Abierta y a Distancia \\ https://orcid.org/0000-0003-4075-2015 \\ Imvaldesc@unadvirtual.edu.co
}

\section{RESUMEN}

Contextualización: La enjambrazón es una de las formas de reproducción natural de las abejas (Apidae: meliponini) que llegan en busca de cavidades preexistentes a formar nuevas colonias que tienden a anidar en lugares atípicos generalmente en áreas suburbanas (viviendas) constituyéndose en blancos para su eliminación por saquear su miel y en otros casos para adquirir sus colonias (nidos); en este mismo sentido se cae en la práctica de talas

DOI: https://doi.org/10.22490/26653176.4289 
o daños parciales a arboles; dejando efectos secundarios en el ambiente natural, estas prácticas muy comunes amenazan con su extinción.

Vació de investigación: Los dispositivos trampa son un señuelo con atrayentes que permiten a las abejas alojarse y formar un nido que posteriormente se trasiegan a colmenas racionales. Las capturas de estas abejas se realizan de manera artesanal, poco técnicas; e incluso validan rupturas en los árboles (técnicas de curación) que terminan en su deterioro y muerte precoz. En particular sobre metodologías de caza enjambres en meliponinos hay escasa información formal, una de las más ajustadas es el uso de trampeos con botellas PETs siendo necesario validar la efectividad de prácticas que contribuyan al crecimiento de poblaciones y no lo contrario.

Objetivo: El objetivo de este trabajo fue validar el método con loción atrayente (geo propóleos más alcohol al 96\%) con tres tratamientos relacionados con la altura de posición de los dispositivos y la cercanía con zonas pobladas en el laboratorio de biodiversidad de la universidad nacional abierta y a distancia UNAD CEAD Acacias.

Metodología: El área de estudio se dividió en 12 cuadrantes, en cada uno de ellos se dispusieron tres dispositivos trampa a diferentes alturas ( $1,1,5$ y 2 metros) donde se describió si el dispositivo se encontraba en un cuadrante cercano o no a un sitio poblado o de construcción para urbanismo; los muestreos se realizaron cada 30 días verificando las capturas y especies.

Resultados y conclusiones: Para el análisis estadístico se utilizó la prueba de chi cuadrado, auxiliado de tablas de contingencia evidenciando un $8,33 \%$ de efectividad de captura de enjambres específicamente de la abeja Tetragonisca angustula, permitiendo un importante resultado comparado con estudios similares que demuestran su efectividad, esto facilita adquirir colonias mediante enjambres para el establecimiento de meliponarios que permitan medir su desempeño, generar conocimiento básico y constituir una base fundamental para el éxito y la inclusión en los sistemas de producción pecuarios.

Palabras Clave: Colonias; Diversidad; Meliponicultura; Trasiego.

\begin{abstract}
Contextualization: Swarming is one of the forms of natural reproduction of bees (Apidae: meliponini) that arrive in search of pre-existing cavities to form new colonies that tend to nest in atypical places, generally in suburban areas (dwellings), becoming targets for their elimination by loot their honey and in other cases to acquire their colonies (nests); in this same sense, the practice of felling or partial damage to trees falls; leaving side effects in the natural environment, these very common practices threaten extinction.
\end{abstract}


Knowledge gap: Trap devices are a lure with attractants that allow bees to lodge and form a nest that are later transferred to rational hives. The captures of these bees are made in an artisanal way, not very technical; and they even validate tree ruptures (healing techniques) that end in their deterioration and early death. In particular, there is little formal information on swarm hunting methodologies in Meliponinos, one of the most appropriate is the use of traps with PET bottles, being necessary to validate the effectiveness of practices that contribute to the growth of populations and not the opposite.

Purpose: The objective of this work was to validate the method with attractant lotion (geo propolis plus $96 \%$ alcohol) with three treatments related to the height of position of the devices and the proximity to populated areas in the biodiversity laboratory of the universidad nacional abierta y a distancia UNAD CEAD Acacias.

Methodology: The study area was divided into 12 quadrants, in each of them three trap devices were arranged at different heights (1, 1.5 and 2 meters) where it was described whether the device was in a quadrant close to a site or not. town or construction for urban planning; the samplings were carried out every 30 days verifying the catches and species.

Results and conclusions: For the statistical analysis, the chi-square test was used, aided by contingency tables, showing $8.33 \%$ effectiveness in capturing swarms specifically of the bee Tetragonisca angustula, allowing an important result compared with similar studies that demonstrate its effectiveness, this facilitates acquiring colonies through swarms for the establishment of meliponaria that allow measuring their performance, generating basic knowledge and constituting a fundamental basis for success and inclusion in livestock production systems.

Keywords: Colonies; Diversity; Meliponiculture; Racking.

\section{INTRODUCCIÓN}

La meliponicultura es el conjunto de técnicas para la cría y manejo de abejas (Apidae: Meliponini) orientadas al aprovechamiento de sus productos. Este término fue propuesto por Nogueira-Neto (1953). De la familia Apidae las abejas de las subfamilias Apinae (presencia aguijón) y Meliponinae (aguijón no funcional) son altamente sociales y poseen colonias perennes que se reproducen por enjambres.

Las abejas (Apidae: Meliponini) construyen sus nidos expuestos o en cavidades preexistentes (huecos de árboles, termiteros, bajo tierra), utilizando cerumen (cera mezclada con resinas), barro y otros materiales (Nates-Parra, 2007). Siendo esto un factor de desventaja. Al igual que son perseguidas por la miel ya que su cosecha es una práctica fácil y sin riegos; esto representa el fin de colonias cuyos nidos lesionados quedan expuestos a hormigas y su principal enemigo, la mosca Phoride que termina acabando con toda la población. 
Un nuevo problema surge a partir del auge de la meliponicultura en nuestra región. Hacerse a una colonia para empezar meliponarios es una verdadera amenaza para su estabilidad en los ecosistemas; prácticas que incluyen capturas en sus medios naturales provocando destrucción de sus hábitats. Hay evidencias en varios documentos donde la práctica de adquirir colonias se realiza mediante captura de colonias silvestres con herramientas como la motosierra directamente desde sus nidos naturales (Montenegro \& Lacayo, 2014; Morales, 2007) captura independiente por campesinos (Genaro \& Lóriga, 2018) y por capturas desde los troncos de los árboles a cajas racionales (López \& Gutiérrez 2016).

Por métodos no invasivos (trampeos), Delgado \& Sobalvarro (2019), reportan un 26\% de capturas en proyectos con comunidades en Nicaragua. Herrera \& Sabogal (2016) en Cundinamarca reportaron 9 capturas en 142 trampas instaladas ( 9 hectáreas) 6,33\% de los géneros T. angustula y Scaptotrigona. Menezes \& Oriental (2015) reportaron en un estudio en Brasil durante 2 años 61 capturas mediante dispositivos trampa. Silva \& Reis (2016) y Gennari (2019), describen el proceso de fabricación de trampas con botellas, secciones de Bambú y tubos de PVC.

La importancia del estudio realizado radica principalmente en dar a conocer esta metodología de dispositivos trampa y sus resultados que constituye una forma racional de adquirir colonias de abejas (Apidae: Meliponini) para el emprendimiento de meliponarios, aprovechando su multiplicación natural (enjambres).

\section{MATERIALES Y MÉTODOS}

Este trabajo se desarrolló en el CEAD de Acacias Meta - Laboratorio de biodiversidad, localizado entre la sede de la Universidad Nacional Abierta y a Distancia y el barrio Villa Manuela, cuyas coordenadas son $04^{\circ} 00^{\prime} 26,5^{\prime \prime}$ 'Norte $73^{\circ} 46^{\prime} 12,0^{\prime}$ 'Sur a una altitud de $574 \mathrm{~m} \mathrm{~s}$ n m. entre los meses de marzo a junio de 2020.

\section{Dispositivos trampa}

Se utilizaron dispositivos trampa similares a los usados por Villas Bôas (2012) y Oliveira et al. (2013). En este caso particular se prepararon 36 botellas PETs de 1,5 litros, loción atrayente propuesta por Gennari (2019), donde se mezclan partes iguales de propóleos y alcohol puro $\left(9^{\circ}\right)$, se agita y se deja estacionar por 30 a 60 días para que se termine de disolver completamente. Estas sustancias contienen aromas (feromonas) que resultan atractivos para abejas exploradoras que están buscando sitios propicios para el establecimiento de un enjambre. Esta solución se usó para impregnar el interior de la botella, las cuales fueron forradas en una primera capa con papel periódico y luego con una bolsa negra calibre $8 \mathrm{~mm}$; a la tapa se le abrió un orificio de 5 a $10 \mathrm{~mm}$.

\section{Instalación y monitoreo de nidos trampa}

Estos dispositivos se distribuyeron en el laboratorio de biodiversidad que es un área boscosa de 20.000 metros cuadrados a una altura de $1,1,5$ y $2 \mathrm{~m}$ en sitios donde el sol no llega 
directamente sujetados a árboles con alambre dulce a distancias entre dispositivos trampa de 3 a 6 metros.

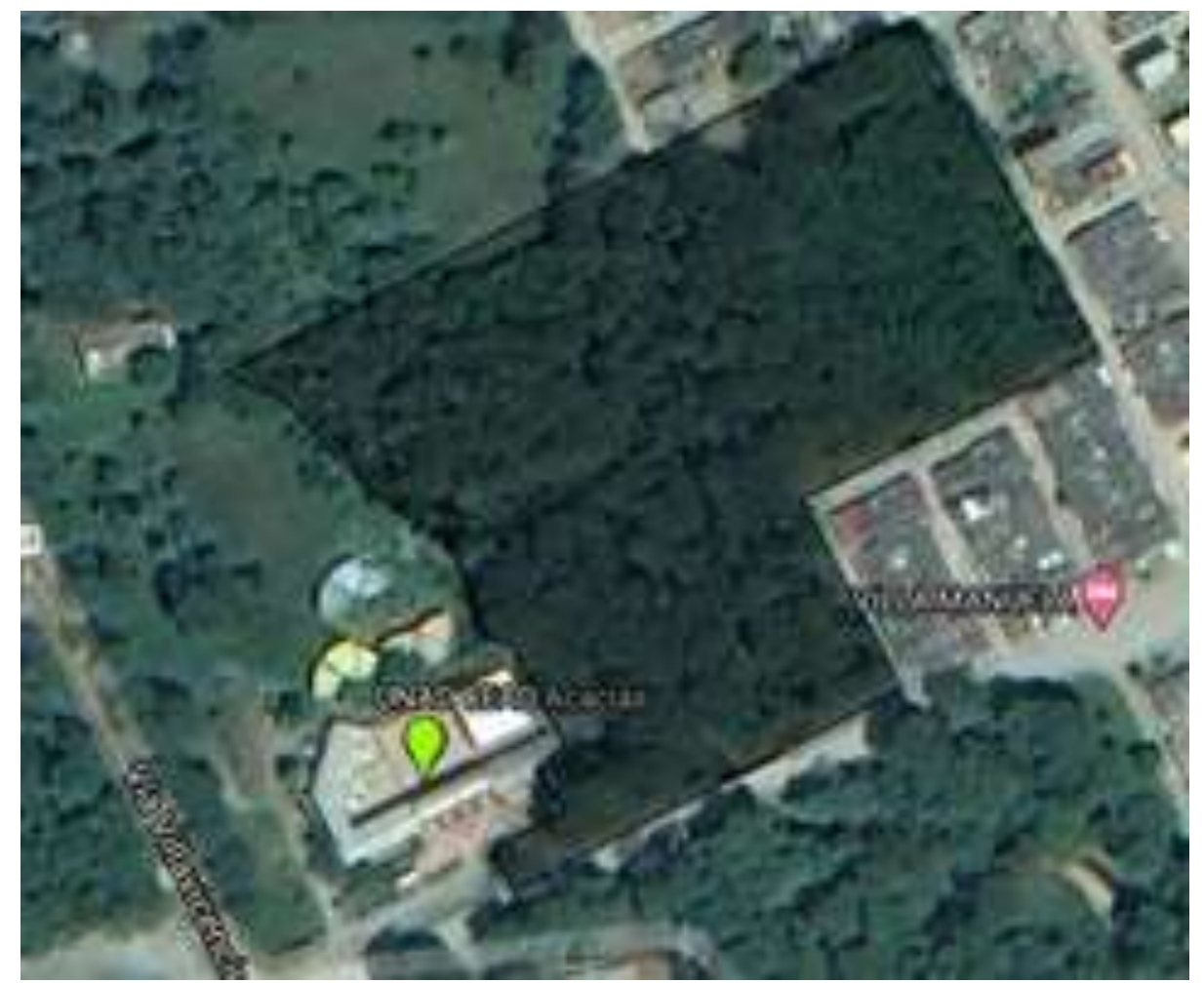

Figura 1. Área de estudio - Laboratorio de biodiversidad - CEAD Acacias. Fuente: autores.

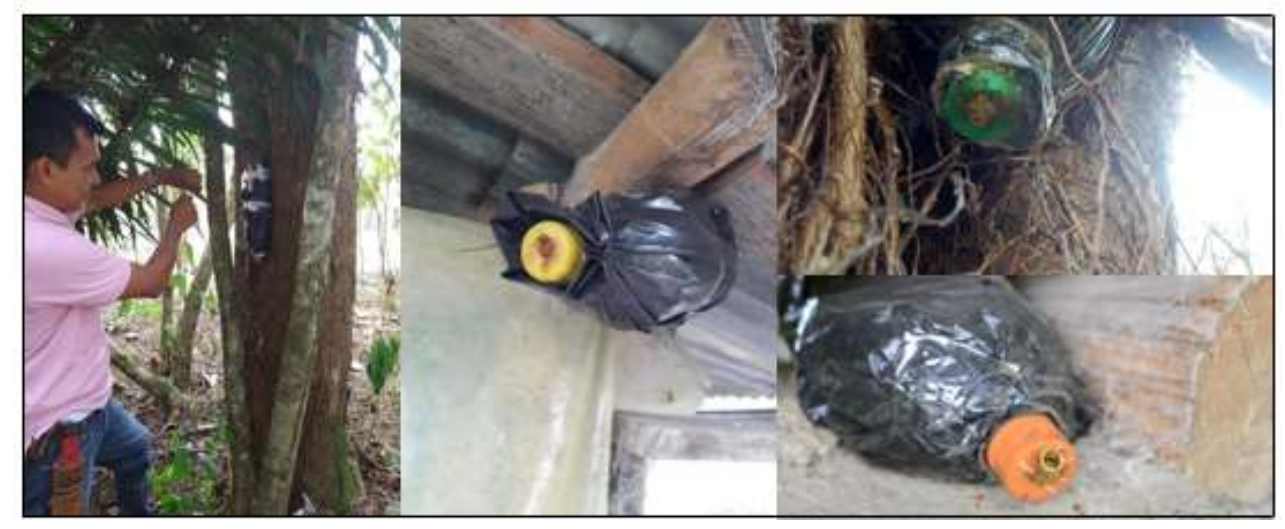

Figura 2. Instalación de trampas PETs - Laboratorio de biodiversidad -CEAD Acacias. Fuente: autores. 


\section{Diseño experimental}

El área boscosa se dividió en doce cuadrantes, en cada uno de ellos se dispusieron tres dispositivos trampa a diferentes alturas (1, 1,5 y 2 metros). Variables consideradas:

- $\quad$ Altura del dispositivo: altura del dispositivo con respecto al suelo.

- Cercanía con las zonas pobladas: (variable nominal - que describe si el dispositivo se encontraba en un cuadrante cercano o no a un sitio poblado o de construcción para urbanismo).

Se realizaron muestreos cada 30 días para verificar si había capturas y poder verificar la especie.

\section{Análisis estadístico}

Se utilizo la prueba de Chi cuadrado con el fin de comparar proporciones, auxiliadas con tablas de contingencia donde se organizaron los datos para cruzar la información y determinar si las variables de estudio estaban relacionadas o no.

La información fue analizada en el programa SPSS de IBM versión 18.0, en el aparte de tablas cruzadas con la indicación de realizar prueba de chi cuadrado, luego de construirse una base de datos con la información relacionada de las variables objeto de estudio.

Como las variables consideradas en el estudio son discretas y nominales se realizó el análisis de la información mediante tablas de contingencia para efectuar una prueba de chi cuadrado, la cual pretendía encontrar la relación entre los factores causales hipotéticos del enjambramiento, tales como la altura de los dispositivos trampa y la cercanía al área poblada. Sin embargo, dicho análisis, no es una prueba de causalidad, demostrándose ciertamente un grado de relación.

Determinación de especie: Se realizó teniendo en cuenta el libro de Delgado \& Rasmussen (2019) donde hay una descripción de las especies (Apidae: Meliponini).

\section{RESULTADOS Y DISCUSIÓN}

\section{Efectividad de los dispositivos trampa}

De los 36 dispositivos trampa que se distribuyeron en el laboratorio de biodiversidad y/o área de estudio, 3 trampas fueron colonizadas por abejas Tetragonisca angustula, correspondiente al $8,33 \%$ de efectividad de captura, datos superiores a los encontrados por Herrera y Sabogal (2016) que obtuvieron un 6,33\% de 142 trampas instaladas capturaron 8 Nidos de 
Tetragonisca angustula y 1 nido de Scaptotrigona. Garzón y Mora (2016) en Guaduas y Caparrapí - Cundinamarca capturaron 5 colonias de 80 trampas instaladas correspondiente al $6,25 \%$ de efectividad.

\section{FACTORES RELACIONADOS CON LA EFECTIVIDAD DEL DISPOSITIVO TRAMPA}

\section{Cercanía a centros poblados o construcciones}

Para analizar lo anterior, en la base de datos se incluyó en cada caso una columna en donde se aclaraba si el dispositivo estaba o no cercano a sitios poblados o construcciones de vivienda.

Todos los dispositivos con enjambre se encontraban cercanos a dichas zonas. (Tabla No. 1).

Tabla 1. Cuadro cruzado de la cercanía y el hecho de enjambre.

\begin{tabular}{ccccc} 
& & \multicolumn{2}{c}{ Enjambre } & Total \\
Cercanía & $\begin{array}{c}\text { No cercano a } \\
\text { poblado }\end{array}$ & 18 & 0 & 18 \\
& $\begin{array}{c}\text { cercanía } \\
\text { construcciones } \\
\text { población }\end{array}$ & 15 & 3 & 18 \\
Total & 33 & 3 & 36 \\
\hline
\end{tabular}

Fuente: autores.

El análisis de chi-cuadrado mostró que efectivamente existe una relación, cuyo nivel de significancia se acercó al establecido inicialmente para el estudio ( $P=0.07)$ (Tabla No. 2).

En otras palabras, no se encontraron enjambramientos en lugares alejados a las zonas pobladas o con construcciones.

Tabla 2. Pruebas de chi-cuadrado cercanía por enjambre

\begin{tabular}{lccc}
\hline & Valor & gl & $\begin{array}{c}\text { Significación } \\
\text { asintótica } \\
\text { (bilateral) }\end{array}$ \\
\hline \multicolumn{1}{c}{ Chi-cuadrado de Pearson } & $3,273^{\mathrm{a}}$ & 1 & 0,070 \\
Corrección de continuidad & 1,455 & 1 & 0,228 \\
Razón de verosimilitud & 4,432 & 1 & 0,035 \\
Prueba exacta de Fisher & 3,182 & 1 & 0,074 \\
Asociación lineal por lineal & 36 & & \\
N de casos válidos & & & \\
\hline
\end{tabular}

Fuente: autores. 


\section{Altura del dispositivo con respecto del suelo.}

En relación a esta variable, la tabla de contingencia No. 3, muestra que ocurrieron 2 enjambramientos en la altura media de $1.5 \mathrm{~m}$ y 1 a la altura de $1 \mathrm{~m}$. No ocurrieron enjambramientos a la altura de 2 metros.

Tabla 3. Cuadro cruzado de la altura y el hecho de enjambre.

\begin{tabular}{lllll}
\hline & & $\begin{array}{l}\text { Enjambre } \\
\text { No enjambre }\end{array}$ & Si enjambre & Total \\
\hline \multirow{3}{*}{ Altura } & $1.00 \mathrm{~m}$ & 11 & 1 & 12 \\
& $1.50 \mathrm{~m}$ & 10 & 2 & 12 \\
Total & $2.00 \mathrm{~m}$ & 12 & 0 & 12 \\
\hline
\end{tabular}

Fuente: autores.

El análisis o prueba de Chi-cuadrado no muestra una relación significativa entre la altura y el hecho de enjambrar $(P=0.336)$. (Tabla No. 4).

Tabla 4. Pruebas de chi-cuadrado altura por enjambre

\begin{tabular}{lccc}
\hline & Valor & gl & $\begin{array}{c}\text { Significación } \\
\text { asintótica } \\
\text { (bilateral) }\end{array}$ \\
\hline Chi-cuadrado de Pearson & $2,182^{\mathrm{a}}$ & 2 &, 336 \\
Razón de verosimilitud & 4,432 & 2 &, 228 \\
Asociación lineal por lineal & 3,182 & 1 &, 466 \\
N de casos válidos & 36 & & \\
\hline
\end{tabular}

Fuente: autores.

El bajo número de enjambramientos (3) y el hecho de que la altura fuera de 3 categorías, explica este resultado, mostrando evidencia no concluyente sobre la independencia de la altura sobre la efectividad del dispositivo.

Los autores consultados, refieren que la eficiencia de captura se debe entre varios factores a:

- Presencia de abejas en el sitio

- Época de instalación de los dispositivos que se realicen cuando haya mayor disponibilidad de flores y pocas lluvias (mayor presencia de enjambres)

- Dispositivos trampa bien fabricados

- Instalación en sitios apropiados (confortables/indirectos al sol)

- Altura adecuada. 
Existen varias metodologías para captura de meliponinos como el "Método CESDA" (Captura de enjambres sin talar el árbol); en el cual resulta inviable ya que en muchos casos el árbol sufre daños que pueden disminuir su vida útil (Coletto- Silva, 2005), y muchas veces muere (Oliveira et al., 2013).

Cuando se capturan de infraestructuras de viviendas algunos recomiendan el método de Monseñor Huberto Bruening descrito por Kerr (1996); que consiste en "transferir el enjambre del hueco a una caja conectada por medio de una manguera". Este método, aunque no implica romper la pared, puede no ser eficiente, ya que depende del paso de la reina a la colmena recién instalada, lo que puede tardar o no suceder.

Por el contrario, el uso de dispositivos trampa contribuyen a una forma eficiente de adquisición de núcleos de abejas meliponas para el inicio de meliponarios, sin causar daños e impactos al medio ambiente.

\section{CONCLUSIONES}

Los dispositivos trampa son efectivos y superiores a los encontrados en referencias de trabajos similares.

La captura de enjambres se relacionó más a la cercanía con los sitios poblados y no con la altura del dispositivo trampa; en este sentido, no se encontraron enjambramientos en lugares alejados a las zonas pobladas o con construcciones. Es de resaltar que las viviendas de este centro urbano son a base de ladrillo hueco lo que hace que varios nidos de estas abejas estén alojados en las construcciones (paredes) y sus enjambres busquen el área de estudio.

En relación a la variable altura del dispositivo ocurrieron 2 enjambramientos en la altura media de $1,5 \mathrm{~m}$ y 1 a la altura de $1 \mathrm{~m}$ y no se evidenciaron enjambres capturados a la altura de 2 metros.

El análisis o prueba de Chi-cuadrado no muestra una relación significativa entre la altura y el hecho de enjambrar.

Crece el acuerdo de metodologías cada vez menos invasivas donde el uso de trampeos permita la obtención de colonias para darle un manejo tecnificado a las abejas meliponas con una interacción positiva entre la producción, la conservación y la extensión agropecuaria.

Es necesario poner el foco en las diversas líneas de investigación para estas especies como lo son: la obtención de colonias para la formación de meliponarios de manera sostenible, instrumentos y accesorios que faciliten su manejo y garanticen un manejo tecnificado para potencializar su conservación y permitirles expresar su potencial de producción.

En la secuencia este tipo de trabajos de captura de enjambres deben permitir el primer paso hacia una meliponicultura racional; que es adquirir el insumo esencial "la colonia de abejas" 
de forma racional y que posteriormente se alojen en colmenas racionales como las descritas por Martínez \& Blandón (2019).

\section{CONTRIBUCIÓN DE AUTORÍA}

El esquema de trabajo se realizó de la siguiente manera:

Primer autor: metodología, diseño experimental, análisis de datos (Estadística)y escritura.

Segundo autor: revisión, edición y escritura colaborativa.

Tercer autor: logística, adquisición de recursos y escritura colaborativa.

\section{AGRADECIMIENTOS}

Los autores agradecen a la Universidad Nacional Abierta y a Distancia CEAD Acacias por el espacio que ha permitido en sus instalaciones para el desarrollo de trabajos con abejas (Apidae: Meliponini) y a los integrantes del Semillero de Investigaciones Pecuarias, Agrostología y Sistemas Silvopastoriles SIPASS por su trabajo en equipo que hicieron posible la materialización de estas ideas.

\section{REFERENCIAS}

Coletto-Silva, A. (2005). Captura de enxames de abelhas sem ferrão (Hymenoptera, Apidae, Meliponinae) sem destruição de árvores. Acta Amazonica, 35(3), 383-388. https://doi.org/10.1590/S0044-59672005000300012

Delgado Vásquez, C., \& Rasmussen, C. (2019). Abejas sin aguijón (Apidae: Meliponini) en Loreto, Perú. Instituto de Investigaciones de la Amazonia Peruana.

Delgado, Y. G. L., \& Sobalvarro, J. A. A. (2019). Proceso de aprendizaje agroecológico desde la práctica de la meliponicultura: una experiencia de campesinos en Santa Lucía, Nicaragua. La Calera, 19(33), 81-87.

Garzón Medina, S., \& Mora Flórez, F. (2016). Evaluación de la Polinización Mediana por Abejas en el Cultivo de Café (coffea arabica) en los Municipios de Guaduas y Caparrapí (Cundinamarca) (Doctoral dissertation).

Gennari, G. P. (2019). Manejo racional de las abejas nativas sin aguijón (ANSA). Ediciones INTA.

Genaro, J. A., \& Lóriga, W. (2018). Melipona beecheii Bennett (Hymenoptera: Apidae): origen, estudios y meliponicultura en Cuba. Insecta Mundi. 
Gennari, G. P. (2019). Manejo racional de las abejas nativas sin aguijón (ANSA). Ediciones INTA.

Herrera Gonzáles, O. V., \& Sabogal Sabogal, J. E. (2016). Evaluación De La Polinización De Café Coffea Arabica Con Abejas Nativas (Apidae: Meliponini) En Un Cultivo Agroecológico En La Mesa-Cundinamarca (Doctoral dissertation).

Kerr, W. E. Carvalho, G. A. Nascimento, V. A. (1996) Abu Uruçu: biologia, gestión y conservación. Minas Gerais: Fundação Acangaú.

López Tenorio, J. D., \& Gutiérrez Galindo, M. (2016). Sistematización de experiencias en Meliponicultura para el mejoramiento del eslabón producción de la cadena productiva en los municipios de Masatepe, Masaya y Yalí, Jinotega. 2014 (Doctoral dissertation, Universidad Nacional Agraria, UNA).

Martínez, D. E. C., \& Blandon, O. J. O. (2019). Meliponario SIPASS-una experiencia con la abeja angelita Tetragonisca angustula con dos tipos de colmenas racionales en el de CEAD Acacias. Documentos de Trabajo ECAPMA, (2).

Menezes, C. (2015). Multiplicación a gran escala de las colonias de abejas sin aguijón para servicios de polinización del sector agrícola Informaciones generales de la institución.

Montenegro, J. D., Balmaceda, L., \& Lacayo, L. (2014). Aporte de la meliponicultura a la economía familiar en El Pochote, El Arenal y Nuevo Amanecer, Masatepe, Masaya, 2013. La Calera, 14(23), 89-95.

Morales, S. M. (2007) Manual de Trigonicultura para la Huasteca Tamaulipeca. México.

Nates-Parra G. (2005) Abejas corniculadas de Colombia. Bogotá:Universidad Nacional de Colombia. p. 156.

Nogueira-Neto P. (1953) La cría de abejas autóctonas sin aguijón (Meliponinae). São Paulo: Ed. Chácaras e Quintais. p. 280.

Silva, F. O. D. y Reis, S. T. D. (2016). Actas de reuniones sobre los beneficios de las abejas en la agricultura.

Oliveira, R. C, Menezes C, Soares A. E, Imperatriz-Fonseca V. L. (2013) Nidos-trampa para abejas sin aguijón (Hymenoptera, Meliponini Apidologie).

Villas-Bôas, J. (2012) Manual tecnológico: abejas sin aguijón. Brasilia, DF: Instituto Sociedad, Población y Naturaleza. (Instituto Sociedad, Población y Naturaleza. Serie de manuales tecnológicos). 